\title{
The Calorific Value of American Woods ${ }^{1}$
}

\author{
By S. W. Parr and C. N. Davidson
}

UNIVERSITY OF ILLINOIS, URBANA, IIIINOIS

W OOD as a fuel may have war-time interest only, or it may be a matter of regional concern or of significance in special industries where wood waste accumulates to a point where it must be disposed of. These conditions may in themselves be a sufficient reason for desiring to know the calorific value of such material. There are other reasons also worth mentioning. At the time this work was carried out (1916), at least, there were no values given in the literature for American woods. Similarly, it is not far wrong to state that there were no values that were worth anything given in the literature for any woods in any country. For example, Count Rumford's values are frequently given in works of reference, ${ }^{2}$ His work was done 110 years ago, ${ }^{3}$ and while he had the correct idea as to the proper method of procedure, his results were little better than approximations. His sample was burned in a current of air and the products of combustion were conducted through a copper coil surrounded by a known volume of water. His thermometer he made himself, but the chief elements of error were doubtless the uncertainty as to the completeness of combustion and the vague ideas concerning the amount or nature of the moisture present in the wood. Again Groves and Thorp, in their work on "Chemical Technology," tabulate the heat values for wood as derived by Berthier, Winkler, and Schödler and Peterson. These values refer to partially dry, well-seasoned wood, but the real content of moisture is uncertain. Worse than this, however, is the fact that all these values were determined by calculation from the weight of a lead button reduced from litharge. The equivalent of oxygen taking part in the reaction is used as a measure of the amount of heat, according to the so-called Welter law which held that a fixed ratio existed between oxygen consumed and heat produced, no matter what combinations were involved. The results obtained do not have the value of a good guess and would need to be increased by about 50 per cent to come even approximately near the true values. Berthier's results were published in 1835 . This date, together with the method employed, suggests the absurdity of repeating them except as curiosities. They do not have as much merit as Rumford's values obtained some 20 years previous, the errors being about twice as great.

In other works of reference as Poole's "Calorimetry" (1903), Brame's text on "Fuels" (1914), etc., the values quoted are those of Gottlieb. ${ }^{5}$ These values are more recent and have the advantage that they were calculated to the oven-dry basis. He used a calorimeter with oxygen as the medium of combustion, but it was of the constant pressure type, which seems not to have been capable of as great refinement in results as should characterize the values which are to be used continuously as a standard of reference. Results obtained 40 years ago with an apparatus which to-day is not recognized as standard should at least be worked over for correction or verification. In Poole's tables, note should be made of

1 Presented before the Division of Industrial and Engineering Chemistry at the 63rd Meeting of the American Chemical Society, Birmingham, Ala., April 3 to $7,1922$.

2 Groves and Thorp, "Chemical Technology. Vol, 1-Fuel and Its Applications," 1889, 338.

${ }^{3}$ Nicholson's Journal, 32 (1812), 105; 35 (1813), 95.

4 "Fuel and Its Applications," 1 (1889), 380.

6 J. prakt. Chem., 28 (1883), 414. two results by Mahler, which doubtless are reliable. They are given as "cinder and water deducted," while the seven values of Gottlieb are not indicated in Poole as to their basis of reference. The original article, however, shows that they refer to dry or "moisture-free" material. The moisturê factor was determined by drying for 12 to $15 \mathrm{hrs}$. in a hot air bath maintained at $115^{\circ}$.

It is evident from this brief survey that the determination of the free moisture present is in as much need of review as the calorific value. Indeed, the latter factor is of little worth without the former. Even greater ambiguity is found in the use of terms than is found in the case of coal. Dry wood is understood to mean well-seasoned wood, and there seems to be a reluctance to recognize $a$ sharp line of demarcation between the free moisture and the very large amount of hydrogen and oxygen chemically combined in the $\mathrm{H}_{2} \mathrm{O}$ ratio $\left(\mathrm{C}_{6} \mathrm{H}_{10} \mathrm{O}_{5}\right)_{x}$ of cellulose.

Our experiments were first directed toward the determination of the "end-point," if there was one, for the free moisture. Was it obscured by uncertain volatilization of sap constituents, or oxidation of unsaturated compounds in the drying process, or possibly an initial breaking down of the cellulose molecule, giving off seemingly free moisture?

Six samples of dry, well-seasoned wood were prepared in the form of fine sawdust. Approximately $1-\mathrm{g}$. samples were heated at $105^{\circ}$ each for 1-, 2-,.3-, 4-, 5-, 6- and 7-hr. periods,

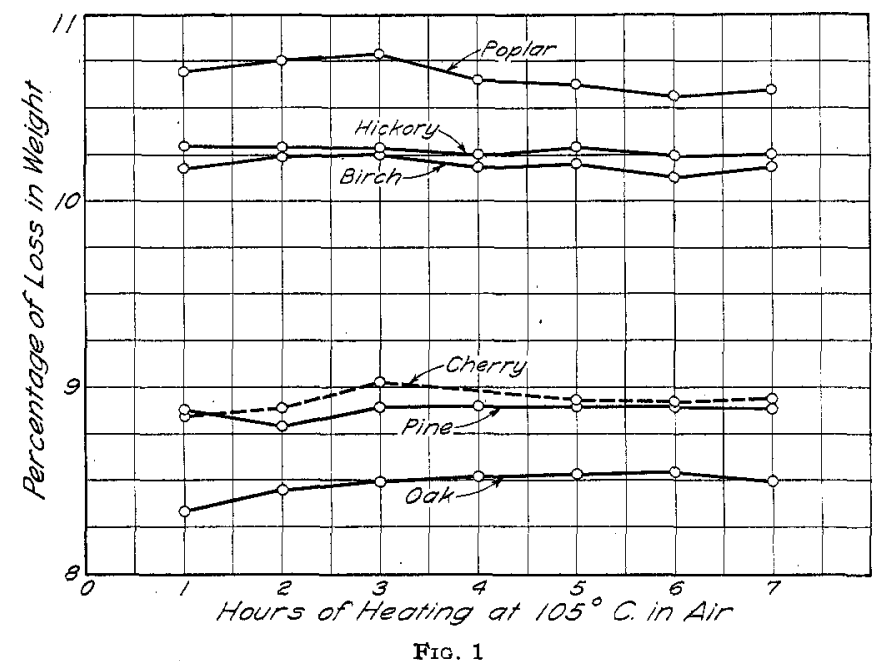

using a new sample for each period and drying in an atmosphere of air and also in carbon dioxide, using a Freas oven with electric heat control. All samples were cooled $20 \mathrm{~min}$. over concentrated sulfuric acid before weighing. The entire series was repeated at $137^{\circ}$, using an atmosphere of air and repeated with carbon dioxide. The curves show interesting results, but only one of each series is given here. It is evident from Fig. 1 that a substantial equilibrium is established after drying in air at $105^{\circ}$ for one hour. A small increase in the air-drying loss is noted up to the end of the third hour. In the use of carbon dioxide as the atmosphere of the oven (not shown) the same percentage of loss is reached at the end of $3 \mathrm{hrs.}$, after which the lines are practically straight. 